\title{
Popularity, Novelty and Attention
}

\author{
Fang $\mathrm{Wu}$ and Bernardo A. Huberman \\ HP Laboratories \\ Palo Alto, CA 94304
}

October 29, 2018

\begin{abstract}
We analyze the role that popularity and novelty play in attracting the attention of users to dynamic websites. We do so by determining the performance of three different strategies that can be utilized to maximize attention. The first one prioritizes novelty while the second emphasizes popularity. A third strategy looks myopically into the future and prioritizes stories that are expected to generate the most clicks within the next few minutes. We show that the first two strategies should be selected on the basis of the rate of novelty decay, while the third strategy performs sub-optimally in most cases. We also demonstrate that the relative performance of the first two strategies as a function of the rate of novelty decay changes abruptly around a critical value, resembling a phase transition in the physical world.
\end{abstract}




\section{Introduction}

As millions of people use the web for their social, informational, and consumer needs, content providers vie for their limited attention by resorting to a number of strategies aimed at maximizing the number of clicks devoted to their web sites [1]. These strategies range from data personalization and short videos to the dynamic rearrangement of items in a given page, to name a few [2, 3, 8]. In all these cases the ultimate goal is the same: to draw the attention of the visitor to a website before she proceeds to the next one [4]. Obviously, the more interesting and relevant the site the more valuable it will be to users. In addition, since users need to decide among the existing plethora of links and sites, their popularities are a determinant of their success, for people often click on given links for no other reason than the fact that many others do. If we add the fact that without novelty attention tends to decay in time, one has a first order list of the requirements for capturing people's attention.

Within this context, we have recently shown that there is a strong interplay between novelty and collective attention, which is universally manifested in a rather swift initial growth of the number of people looking at a new item within a site and its eventual slowdown as interest fades among the population [7]. This result suggests that ordering the links of a given page by their novelty can guarantee a high degree of attention. This is indeed the case in many news websites, notably digg. com.

And yet, given the role that popularity plays in attracting the attention of users, a natural question arises as to whether alternative orderings, like one giving priority to popularity over novelty, might not do better at attracting viewers to a site.

This paper answers this question by taking the dynamics of collective attention to a finer level of detail and examining the role that popularity and novelty play in determining the number of clicks within a given page. In particular, we study three different strategies that can be deployed in order to maximize attention. The first strategy prioritizes novelty while the second emphasizes popularity. The third strategy looks myopically into the future and prioritizes stories that are expected to generate the most clicks in the next few minutes. We 
show that the first two strategies should be selected on the basis of the rate of novelty decay, while the third strategy performs sub-optimally in most cases. Most interestingly, we discover that the relative performance of the first two benchmark strategies as a function of the rate of novelty decay switches so sharply around some critical value that it resembles phase transitions observed in the real world.

The work is organized as follows. We first study the question of whether or not the location of a link in a page determines the overall number of clicks in a given time interval. Having answered this in the affirmative through an empirical study of digg.com, we then proceed to introduce a set of indexes whose values determine the optimal strategy to be pursued in order to maximize attention to a page. Using measured values of the rate of decay from digg.com we built a realistic simulator to collect statistically significant data to measure each of the indices introduced.

We then study the performance of each of these indices as a function of the decay rate and show which strategy optimizes viewing for given values of the decay. Most importantly we compute a full phase diagram that indicates at a glance the optimal strategy to use given the parameter values of the site. This phase diagram exhibits a sharp boundary between the choice of prioritizing novelty over popularity, thus resembling a phase transition.

Finally we summarize our results and discuss their implications for the design of dynamic websites.

\section{Location matters}

In this section we study how the order in which links are placed within a webpage (e.g. the news stories of digg.com) determines the number of clicks within a certain time frame. Assume that time flows discretely as $t=0,1,2 \ldots$ minutes. Let $N_{t}$ denote the number of clicks, or digg number of a story in digg. com, that appeared on the website $t$ minutes ago (in this case we say that the story has lifetime $t$ ). As we showed earlier [7] the growth of $N_{t}$ satisfies the following stochastic equation:

$$
N_{t+1}=N_{t}\left(1+a r_{t} X_{t}\right)
$$


where $r_{t}$ is a novelty factor that decays with time and satisfies $r_{0}=1$, $X_{t}$ is a random variable with mean 1 , and $a$ is a positive constant.

This equation takes into account two important factors that together determine the growth of collective attention: popularity and novelty. The popularity effect is captured by the multiplicative form of Eq. (1), and the novelty effect is described by $r_{t}$. All other factors are contained in the noise term $X_{t}$.

We next take the analysis to a finer level by considering a third position factor. A news story displayed at a top position on the front page easily draws more attention than a similar story placed on later pages. Hence the growth decay $a r_{t}$ should depend on the physical position at which the story is posted.

In the specific case of digg.com, its front page is divided into 15 slots, being able to display 15 stories at a time. The stories are always sorted chronologically, with the latest story at the top. If we label the positions from top to bottom by $i=1,2, \ldots, 15$, we can modify Eq. (11) to allow for an explicit dependency of $a$ on $i$ :

$$
N_{t+1}=N_{t}\left(1+a_{i} r_{t} X_{t}\right)
$$

where $a_{i}$ is a position factor that decreases with $i$.

The assumption that the novelty effect and the position effect can be separated into two factors $r_{t}$ and $a_{i}$ needs to be tested empirically. To this end we tracked the growth rate for each slot, rather than for each story. For multiplicative models it is convenient to define the logarithmic growth rate

$$
s_{t}=\log N_{t+1}-\log N_{t} .
$$

When $a$ is small (which is always true for short time periods) we have from Eq. (2)

$$
s_{t}^{i} \approx a_{i} r_{t} X_{t}
$$

for a story placed at position $i$ at time $t$. Taking expectation of both sides, we have

$$
E s_{t}^{i} \approx a_{i} r_{t}
$$

since $E X_{t}=1$.

The logarithmic growth rate $s_{t}^{i}$ can be measured as follows. For each fixed position $i$, if a digg story appears on that position at both 


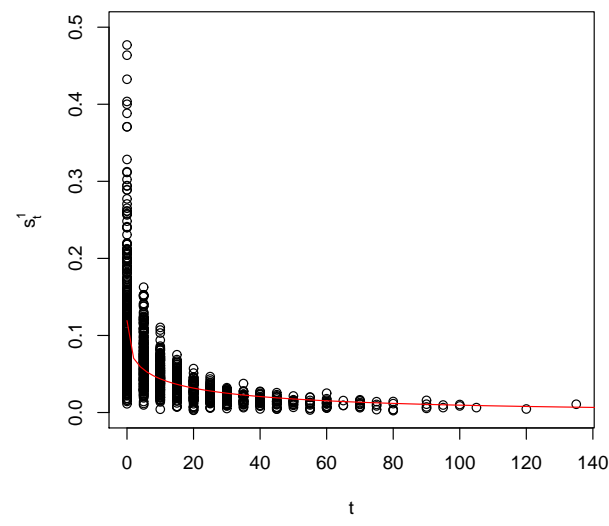

(a)

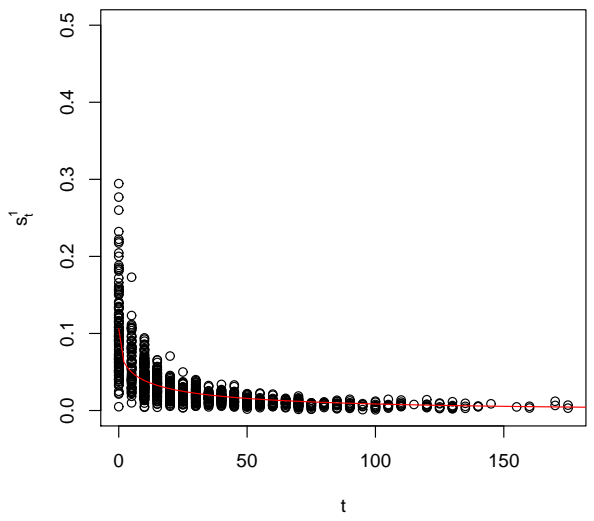

(b)

Figure 1 - The logarithmic growth rate for the top two positions on the front page of digg.com. Time is measured in minutes. Data is collected every 5 minutes, the rate at which the front page is refreshed. The solid curve in (a) is the result of a minimum mean square fit to the data (see text for more details). It has the functional form $f(t)=0.120 e^{-0.4 t^{0.4}}$. The curve in (b) has the functional form $f(t)=$ $0.106 e^{-0.4 t^{0.4}}$.

times $t$ and $t+5$ (the front page is refreshed every 5 minutes), then the observed quantity $\frac{1}{5}\left(\log N_{t+5}-\log N_{t}\right)$ counts as one sample point of $s_{t}^{i}$. Fig. 1(a) plots 1,220 sample points collected from the top position at various times. Fig. 1(b) is a similar plot for the second top position. By comparing (a) and (b) we see that $s_{t}^{2}$ indeed tends to fall below $s_{t}^{1}$, which indicates that the position effect is real. To better illustrate the position effect, we plot the expected growth rate for position 1, 3 and 5 in Fig. 2. As can be seen there, the growth rate decays as the story moves to lower positions.

From this data we can also determine the values of $a_{i}$ quantitatively. We already established that for digg.com the precise functional form of the decay factor is $r_{t}=e^{-0.4 t^{0.4}}$. Thus, for these particular 


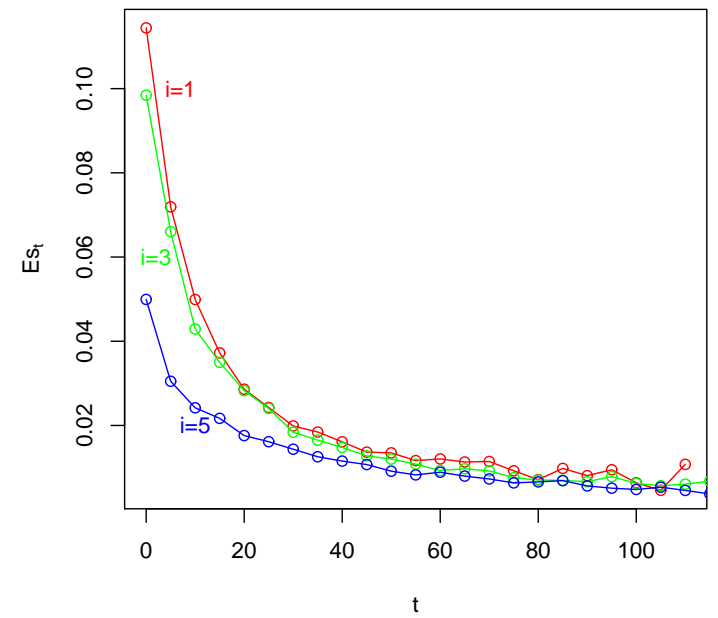

Figure $\mathbf{2}$ - The expected logarithmic growth rate for position 1, 3 and 5 on the front page of digg.com. Time is measured in minutes. As can be seen, the growth rate decays as the story moves to lower positions.

values, the minimum mean square estimator $\hat{a}^{i}$ minimizes

$$
\min _{a^{i}} \sum_{j}\left[s_{t_{j}}^{i}(j)-a^{i} r_{t_{j}}\right]^{2}=\min _{a^{i}} \sum_{j}\left[s_{t_{j}}^{i}(j)-a^{i} e^{-0.4 t_{j}^{0.4}}\right]^{2},
$$

where $t_{j}$ is the lifetime of the $j$ 'th data point. The estimator for the 1,220 data points obtained from the top position is calculated to be $\hat{a}^{1}=0.120$. The fitted curve $\hat{a}^{1} r_{t}=0.120 e^{-0.4 t_{j}^{0.4}}$ is shown as a solid curve in Fig. 1(a). An estimator $\hat{a}^{2}=0.106$ for the second top position is also calculated and plotted in Fig. 1(b). As can be seen from those figures, the position effect $\left(a^{i}\right)$ and the novelty effect $\left(r_{t}\right)$ can indeed be separated. We can then conclude that Eq. (2) fits the data very well. 


\section{Optimal ordering for maximal at- tention}

We now consider the order in which news stories should be displayed on a web page so as to generate the largest number of clicks within a certain time period $T$. This time period needs to be finite because the total number of clicks diverges as $T$ goes to infinity. Equivalently, in an infinite-horizon framework, we could discount future clicks with a discount parameter $\delta$, so that one click at time $t$ counts as $\delta^{t}$ click at time 0 . The objective then is to maximize $\sum_{t=0}^{\infty} \delta^{t} N_{t}$, where $N_{t}$ is the total number of clicks generated from the news page in period $t$. In what follows we will consider the finite-horizon objective.

To simplify the problem we confine ourselves to a subset of ordering strategies called indexing strategies, which is defined as follows. Given a story's state, which in our model is just a two-vector $\left(N_{t}, t\right)$, one first calculates an index $O$ for each story using a predefined index function $O\left(N_{t}, t\right)$, and then sorts the stories based on their indices. The story with the largest index is displayed at the top, the story with the second largest index next, and so on [5, 6].

Rather than considering a general index function we will concentrate on three simple strategies. While neither of them is perfect, each can increase overall attention to the site.

1. $O_{1}(t)=-t$. The stories are sorted by their novelty, with the newest story at the top. This is what digg.com is doing today.

2. $O_{2}(t)=N_{t}$. The stories are sorted by their popularity, with the most popular story at the top. This strategy is based on the fact that attention grows in a multiplicative fashion (popular stories are more likely to become even more popular).

3. $O_{3}(t)=N_{t} r_{t}$. This is the "one-step-greedy" strategy. Ignoring the position effect (assume $a=1$ ), a story in state $\left(N_{t}, t\right)$ generates on average $N_{t} r_{t}$ more clicks (or "diggs" if one considers digg. com) in the next period. This strategy thus places the most "replicated" story at the top.

Notice that because $N_{t}$ grows with time, the effect of sorting by $O_{1}$ is almost the opposite of sorting according to $\mathrm{O}_{2}$. 
In order to test these strategies, we built a simulator that closely resembles the functioning of digg.com in that it incorporates the following rules:

1. Initially there are 15 stories, all in state $\left(N_{t}, t\right)=(1,0)$. In words, each story starts with 1 digg and lifetime 0 . (Because our model is purely multiplicative, the initial digg number does not matter. We just set it to be 1.)

2. Allocate the 15 stories to 15 positions, in decreasing order of their $O\left(N_{t}, t\right)$, for any given index function $O$.

3. Time evolves one step ( 5 minutes) at a time. The number of diggs generated from a story at position $i$ is given by

$$
\Delta N_{t+5}=N_{t+5}-N_{t}=5 a_{i} r_{t} X_{t} N_{t}
$$

The total number of diggs generated in this time step is the sum of 15 such numbers.

The values of $a_{i}$ were estimated from real data and shown in Fig. 3. $r_{t}=e^{-0.4 t^{0.4}} . \quad X_{t}$ is randomly drawn from a normal distribution with mean 1 and standard deviation 0.5 (obtained from the real data from digg.com).

4. On average every 20 minutes a new story arrives. Thus the number of stories arriving in one time step ( 5 minutes) follows a Poisson distribution with mean 0.25 . When a new story enters the pool, the story with the lowest index is dropped, maintaining 15 stories in total. (It is possible the a new story is dropped immediately after its arrival if it happens to have the lowest index.)

5. Go back to Step 2 until the loop has been repeated for enough rounds.

The performance of all three index functions were tested in our simulator. For each index function, Steps 2 to 5 were repeated 100,000 times (or equivalently 500,000 minutes). Strategy $O_{1}$ (sort by novelty) achieved a total number of 514,314.8 diggs. Strategy $\mathrm{O}_{2}$ (sort by popularity) only generated 354.6 diggs. Strategy $O_{3}$ (one-step-greedy) generated 452,402.3 diggs. Thus for these parameter values $O_{1}$ turns 


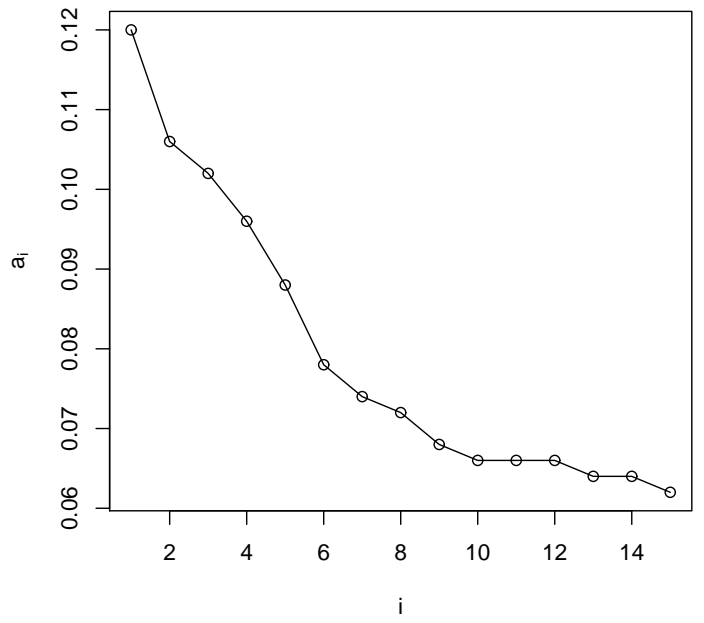

Figure 3 - The position factor decays as the position lowers. The values of $a_{i}$ are measured by tracking the 15 slots on digg.com's front page.

out to be best strategy, since it is $13.7 \%$ better than $O_{3}$ and tremendously better than $\mathrm{O}_{2}$. This confirms that digg.com is using the right strategy.

The reason for the poor performance of the index $\mathrm{O}_{2}$ is easy to understand. $\mathrm{O}_{2}$ gives higher priority to stories that have been dugg many times. According to the indexing rule, after one period new stories can never find their way to the front page since all the old stories have more than 1 digg! When novelty decays fast, the old stories remaining on the front page soon lose their freshness and cease to generate any new diggs. The system thus gets frozen in an unfruitful state.

The fact that $O_{1}$ outperforms $O_{3}$ is a bit harder to understand. Some intuition can be gained by considering an extreme case. Suppose each story completely loses its novelty after one second $\left(r_{0}=1, r_{t}=0\right.$ for all $t>0$ ). Then only "new arrivals" should be displayed since they are the only ones that can generate new diggs. Sorting stories by their lifetime is a good idea when novelty decays fast. On the other hand, if 
novelty never decays $\left(r_{t} \equiv 1\right)$, the lifetime factor becomes irrelevant. Thus in this case, strategy $\mathrm{O}_{3}$, which prioritizes popular stories, will win over $O_{1}$. Hence, the fact that $O_{1}$ works better than $O_{3}$ in our simulations shows that novelty decays relatively fast for digg.com. Should it decay at a slower rate, $O_{3}$ would be a better choice.

We point out that our simulation only showed that the ordering implied by $O_{1}$ works better than $O_{3}$ for a particular choice of $T$. In general this may not be true for other values of $T$. In fact, for a time interval of $T=5$ minutes (one time step) $O_{3}$ is by definition the best strategy. Hence, comparing the performance of two or more index functions only makes sense after one has specified a time horizon (or how much the future should be discounted if an infinite horizon is assumed).

In order to quantitatively test the limiting behavior of the three strategies, we repeated our simulations for a range of different values of the decay parameter $r_{t}$. Our previous work suggested that $r_{t}$ decays as a stretched exponential function, whose general form can be written as $r_{t}=e^{-\alpha t^{\beta}}$. For digg.com it turns out $\alpha=\beta=0.4$. The parameter $\beta$ determines the decay rate. For fixed $\alpha$, the larger $\beta$, the faster $r_{t}$ decays. We repeated our experiment for $\alpha=0.4$ and $\beta \in[0.30,0.45]$. The result is shown in Fig. 4. The performance of each indexing strategy is measured by the logarithm of the total number of diggs generated in 10,000 rounds. We see that as $\beta$ increases (faster decay), the number of diggs decreases for all three indexing strategies. When $\beta>0.34, O_{1}$ performs slightly better than $O_{3}$ and much better than $\mathrm{O}_{2}$. When $\beta<0.33$, however, $\mathrm{O}_{3}$ and $\mathrm{O}_{2}$ perform significantly better than $O_{1}$. In other words, on the two sides of the value of $\beta=0.335$, the stories should be displayed in completely reversed order! We therefore say that a phase transition takes place at the value of $\beta=0.335$.

Other points worth mentioning are that in Fig. $4 O_{3}$ asymptotically approaches $O_{1}$ and $O_{2}$ both in the fast and slow decay limits, and that in general $\mathrm{O}_{3}$ is the best index among the three strategies (although for the specific parameters of digg. $\operatorname{com}(\alpha=\beta=0.4)$ and our particular time horizon $O_{1}$ is slightly better). This is because $O_{3}$ trades off between popularity and novelty instead of betting on only one factor. 
To see this, consider the equivalent index function

$$
O_{3}^{\prime}\left(N_{t}, t\right)=\log O_{3}\left(N_{t}, t\right)=\log N_{t}+\log r_{t} .
$$

Clearly, $O_{3}^{\prime}$ linearly trades off between $\log N_{t}$ and $\log r_{t}$, assigning identical weight to the two effects. This is by no means the best tradeoff. For example, the index function

$$
O_{4}\left(N_{t}, t\right)=0.6 \log N_{t}+\log r_{t}
$$

achieves 556,444.1 diggs after 100,000 rounds of simulation, which is $8.2 \%$ more than $O_{1}$ and $23.0 \%$ more than $O_{3}$ ! However arbitrary it may seem to give the term $\log N_{t}$ weight 0.6 rather than 1 is beyond the scope of this paper, but it does show the complexity of our problem. These experiments demonstrate that the novelty decay rate needs to be measured with great care, as a slight change in the decay rate may totally reverse the optimal order needed to maximize attention.

It is usually hard to analytically compute the performance of a general index function. For the two simple strategies $O_{1}$ and $O_{2}$, however, some rough estimate can be achieved. For the sake of generality, assume that there are $m$ positions on the front page. New stories arrive at a rate $\lambda>0$. Novelty decays as $r_{t}=e^{-\alpha t^{\beta}}$, where $0<\beta \leq 1$. Let $\bar{a}=\frac{1}{m} \sum a_{i}$ be the average position factor, which equals 0.08 for digg.com. Let $\Delta t$ be the refresh time step, which is 5 minutes for digg. com.

Consider strategy $\mathrm{O}_{2}$ first. According to the index rule, new stories never appear on the front page. All diggs are generated by the initial $m$ stories. After time $T$ we have from Eq. (3) that

$$
\log N_{T}=\sum_{t=0, \Delta t, \ldots, T-\Delta t} a_{i} r_{t} X_{t} \Delta t
$$

Hence on average each story's log-performance is

$$
E \log N_{T}=\sum_{t=0, \Delta t, \ldots, T-\Delta t} \bar{a} r_{t} \Delta t \approx \bar{a} \int_{0}^{T} r_{t} d t .
$$

When $T$ is large, we have

$$
E \log N_{T} \approx E \log N_{\infty}=\bar{a} \int_{0}^{\infty} r_{t} d t .
$$




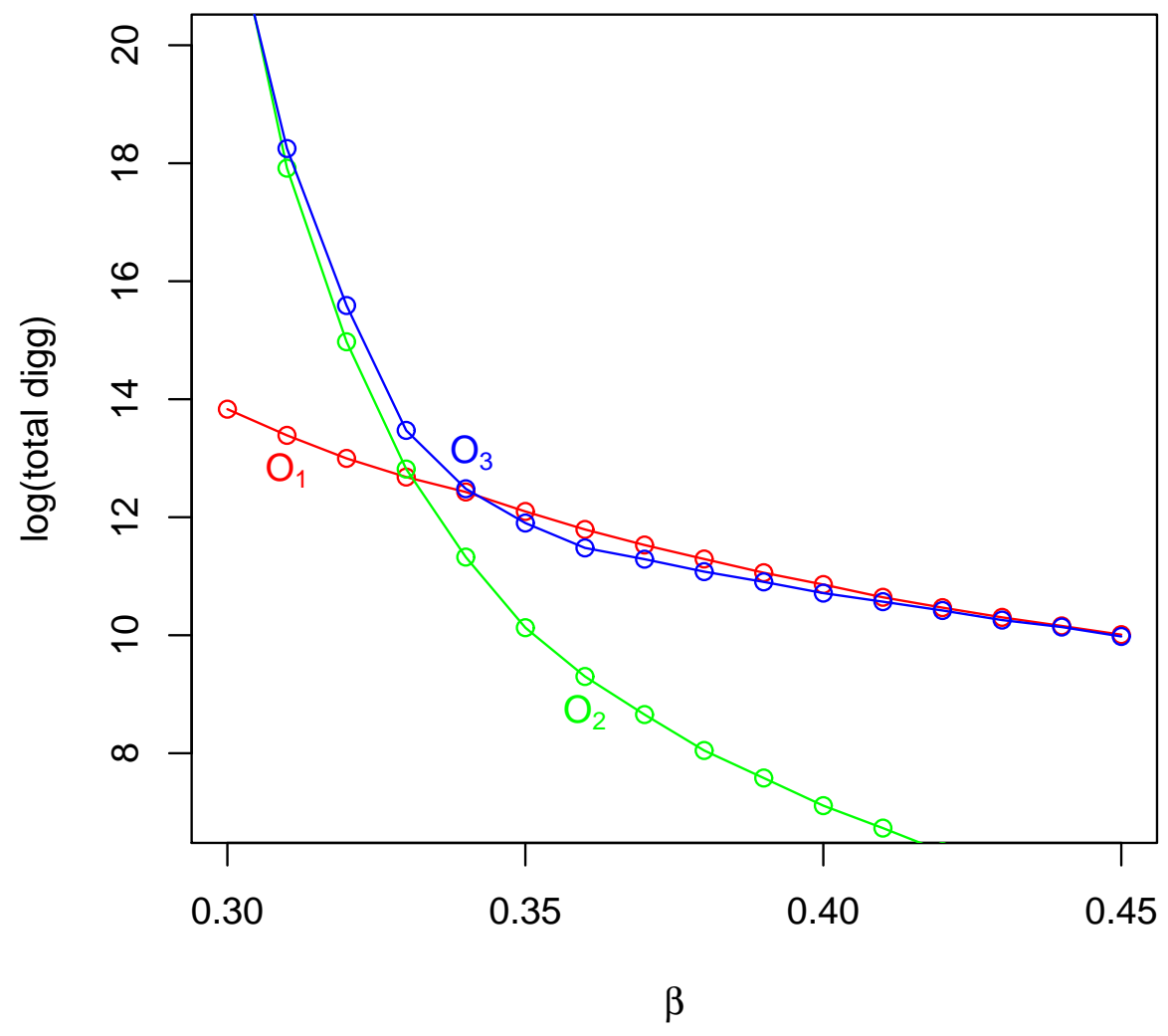

Figure 4 - The total number of diggs generated using three ordering strategies $O_{1}, O_{2}$, and $O_{3}$, for $\alpha=0.4$ and a range of $\beta$. The novelty factor decays as $r_{t}=e^{-\alpha t^{\beta}}$. Performance is measured by the logarithm of the total number of diggs generated in 10,000 time steps. As can be seen, $O_{3}$ asymptotically approaches $O_{1}$ and $O_{2}$ in the fast decay (large $\beta$ ) and slow decay (small $\beta$ ) limit, respectively. A phase transition happens around $\beta=0.335$. 
Next consider $O_{1}$, which orders the stories by their lifetime. On average every $s \equiv 1 / \lambda$ minutes a new story replaces an old story, and each old story moves down one position. Hence on average each story stays on the front page for $m s$ minutes, where $m$ is the number of positions. We call $m s$ one page cycle. It is the average time it takes to refresh the whole page. We now see that, before a story disappears from the front page, it generates

$$
N_{m s}=\exp \left(\sum_{t=0, \Delta t, \ldots, m s-\Delta t} a_{i(t)} r_{t} X_{t} \Delta t\right)
$$

diggs, where $i(t)$ is the story's position at time $t$. When an story gets replaced by a new story, they are counted as one story restarting from the state $N_{t}=1$ and $t=0$. The multiplicative process starts over, and another $N_{m s}$ diggs are generated in the next $m s$ minutes, on average. Thus, in a total time period $T$ the process is repeated $T /(\mathrm{ms})$ times, and a total number of $N_{m s} T /(m s)$ diggs are generated per story. The log-performance of $O_{1}$ is approximately

$$
\log N_{m s}+\log \left(\frac{T}{m s}\right)=\sum_{t=0, \Delta t, \ldots, m s-\Delta t} \bar{a} r_{t} X_{t} \Delta t+\log \left(\frac{T}{m s}\right),
$$

where we replaced $a_{i}(t)$ by $\bar{a}$ since on average each story stays in position $1, \ldots, m$ for equal times. Taking expectation on both sides, we have

$$
E \log N_{m s}+\log \left(\frac{T}{m s}\right) \approx \bar{a} \int_{0}^{m s} r_{t} d t+\log \left(\frac{T}{m s}\right) .
$$

The critical point can be determined by equating Eq. (12) and (15):

$$
E \log N_{T}-E \log N_{m s}=\log T-\log (m s)
$$

or

$$
\bar{a} \int_{m s}^{\infty} r_{t} d t=\log \left(\frac{T}{m s}\right),
$$

which holds for any functional form of $r_{t}$. The left side of Eq. (16) can be interpreted as the total novelty left after a time $m s$, or the total logperformance that can be gained from one story after one page cycle. The right hand side of Eq. (16) is the total log-time left after one page 
cycle. Thus, Eq. (16) and (17) say that, after one page cycle, if there is more novelty left than the log-time remained, the stories should be ordered by decreasing popularity rather than by decreasing novelty $\left(O_{2}\right.$ is better than $\left.O_{1}\right)$. Conversely, if novelty decays too fast (not enough novelty left after one page cycle), then the stories should be ordered by decreasing novelty rather than decreasing popularity $\left(O_{1}\right.$ is better than $\mathrm{O}_{2}$ ).

When $r_{t}=e^{-\alpha t^{\beta}}$ it holds that

$$
\int_{m s}^{\infty} r_{t} d t=\frac{\alpha^{-\frac{1}{\beta}}}{\beta} \Gamma\left(\frac{1}{\beta}, \alpha(m s)^{\beta}\right),
$$

where

$$
\Gamma(a, x)=\int_{x}^{\infty} t^{a-1} e^{-t} d t
$$

is the incomplete Gamma function. In this case the critical equation can also be written as

$$
\bar{a} \frac{\alpha^{-\frac{1}{\beta}}}{\beta} \Gamma\left(\frac{1}{\beta}, \alpha(m s)^{\beta}\right)=\log \left(\frac{T}{m s}\right) .
$$

For the parameters of digg.com $(\bar{a}=0.08, m=15, s=20)$ and horizon $T=50,000$ one can solve for the critical curve $(\alpha, \beta)$ on which $O_{1}$ and $O_{2}$ have the same performance. The curve is shown in Fig. 5 as a phase diagram. When the parameters $(\alpha, \beta)$ lie above the critical curve, the stories should be sorted by $O_{1}$. Otherwise they should be sorted by $\mathrm{O}_{2}$.

To illustrate how sharp the phase transition is, we plot the relative performance $O_{2} /\left(O_{1}+O_{2}\right)$ as a function of $\beta$, for fixed $\alpha=.4$, in Fig. 6. As can be seen, the transition is indeed very sharp.

\section{Conclusion}

In this paper we have shown that depending on the rate of decay of novelty, two different strategies can be deployed in order to maximize attention. The first one prioritizes novelty while the second emphasizes popularity. Most interestingly, the shift from one to the other as a function of the rate of decay is extremely sharp, resembling the phase transitions observed in the physical world. 


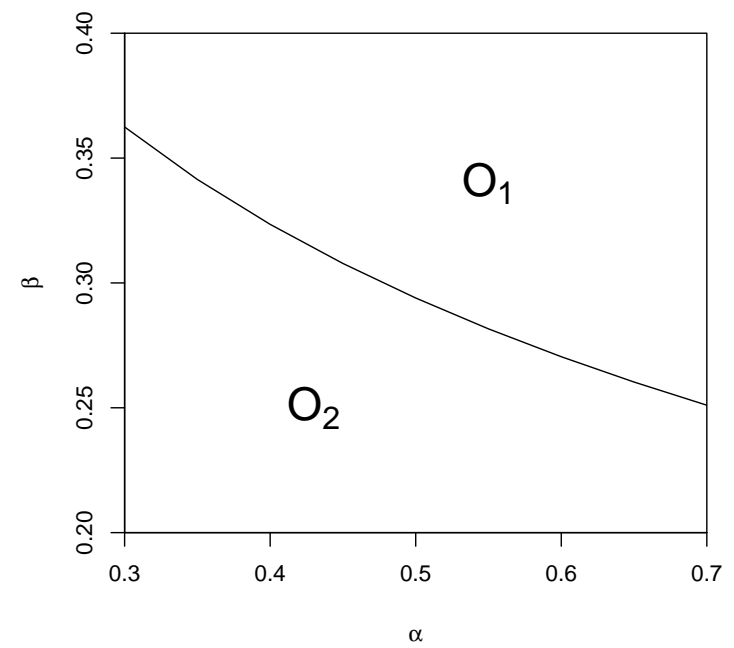

Figure 5 - The phase diagram. The critical curve is calculated by solving Eq. (20) with $\bar{a}=0.08, m=15, s=20$ and $T=50,000$. When $(\alpha, \beta)$ lies in the upper half of the phase diagram $O_{1}$ works better than $\mathrm{O}_{2}$. Otherwise $\mathrm{O}_{2}$ works better.

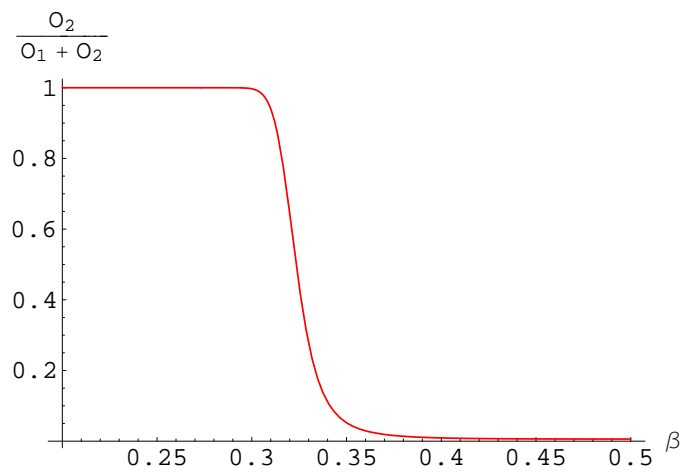

Figure 6 - The relative performance $O_{2} /\left(O_{1}+O_{2}\right)$ as a function of $\beta$, for fixed $\alpha=.4$. 
These results were obtained by focusing on the dynamics of collective attention and examining the role that popularity and novelty play in determining the number of clicks within a given page. In particular, we analyzed three different strategies that can be deployed in order to maximize attention. The first strategy prioritizes novelty while the second emphasizes popularity. The third strategy looks myopically into the future and prioritizes stories that are expected to generate the most clicks in the next few minutes. We then showed that the first two strategies should be selected on the basis of the rate of novelty decay, while the third strategy performs sub-optimally in most cases. Most interestingly, we discovered that the relative performance of the first two benchmark strategies as a function of the rate of novelty decay switches so sharply around some critical value that it resembles phase transitions observed in the real world.

Given the importance of maximizing page views for most content providers, this work suggests a principled way of choosing what to prioritize when designing dynamic websites. Knowledge of the rates with which novelty and popularity evolve within the website can then be translated into decisions as to what to show first, second, etc.

\section{References}

[1] Josef Falkinger. Attention economies. Journal of Economic Theory, vol. 133, pp. 266-294, 2007.

[2] J. Garofalakis, P. Kappos and D. Mourloukos. Web site optimization using page popularity. IEEE Internet Computing, volume 3, issue 4, pp. 22-29, 1999.

[3] Weiyin Hong, James Y. L. Thong and Kar Yan Tam. Does animation attract online users attention? The effects of Flash on information search performance and perceptions. Information Systems Research, vol. 15, no. 1, pp. 60-86, 2004.

[4] Bernardo A. Huberman, Peter L. T. Pirolli, James E. Pitkow, and Rajan M. Lukose. Strong regularities in World Wide Web surfing. Science, vol. 280, no. 5360, pp. 95-97, 1998. 
[5] José Niño-Mora. Stochastic scheduling. Encyclopedia of Optimization, C. A. Floudas and P. M. Pardalos, eds., vol. V, pp. 367-372, 2001.

[6] Fang Wu and Bernardo A. Huberman. The economics of attention: Maximizing user value in information-rich environments. The First International Workshop on Data Mining and Audience Intelligence for Advertising (ADKDD'O7), 2007.

[7] Fang Wu and Bernardo A. Huberman. Novelty and collective attention. Proceedings of National Academy of Sciences, vol. 104, no. 45, pp. 17599-17601, 2007.

[8] Ping Zhang. The effects of animation on information seeking performance on the World Wide Web: Securing attention or interfering with primary tasks? Journal of the AIS, volume 1, issue 1, 2000 . 